\title{
Treatment of Hepatitis C with Sofosbuvir, Velpatasvir and Voxilaprevir Decreases Hemoglobin A1c and Dependence on Anti-Glycemic Medications
}

\author{
Kashif Aziz*1, Mehreen Khan², Amir Shahbaz ${ }^{1}$, Muhammad Umair ${ }^{1}$ and Issac Sachmechi ${ }^{1}$ \\ ${ }^{1}$ Department of Medicine, Icahn School of Medicine Mount Sinai, Queens Hospital Center, USA \\ ${ }^{2}$ School of Medicine and Public Health, the George Washington University School of Medicine, USA
}

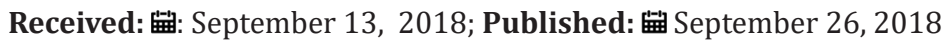

*Corresponding author: Kashif Aziz, Department of Medicine, Icahn School of Medicine Mount Sinai, Queens Hospital Center, Jamaica, New York, USA

\section{Abstract}

Aim: Hepatitis treatment with direct acting antivirals improves markers of diabetes like $\mathrm{HbA1} \mathrm{c}$ and also decreases daily requirement of antidiabetic medications.

Background: Insulin resistance and Type 2 diabetes are one of manifestation of Hepatitis C (HCV). It is proposed that circulating micro ribonucleic acid (miRNA) can do regulatory functions to modulate gene expression in peripheral tissues leading to insulin resistance and diabetes. Clearance of HCV with direct-acting antivirals (DAAs) significantly decreases hemoglobin A1c (HbA1c) and the dependence on anti-glycemic medications.

Case Description: we present a case of HCV positive diabetic patient treated with the combination of sofosbuvir/velpatasvir/voxilaprevir, lead to improvement of his HbA1c as well as significant reduction of his daily insulin dose.

Conclusion: It is concluded that treatment of HCV with DAAs may cause improvement of glucose metabolism and decreases dependence on anti-diabetic medications.

Keywords: Hepatitis C; Sofosbuvir or Velpatasvir or Voxilaprevir; Hba1c; Type 2 Diabetes; Direct Acting Antiviral Agents

Abbreviations: HbA1c: Hemoglobin A1c; DAAs: Direct Acting Antivirals; HCV: Hepatitis C Virus; miRNA: Micro Ribonucleic Acid; CHC: Chronic Hepatitis C Virus Infection; SOF: Sofosbuvir; LED: Ledipasvir; Low LDL: Density Lipoprotein; SVR: Sustained Virologic Response

\section{Background}

Hepatitis C virus (HCV) infection is associated with increased rates of glucose abnormalities, including diabetes mellitus and insulin resistance. The presence of glucose abnormalities in HCV infected patients, including diabetes mellitus and insulin resistance, is associated with negative liver-related outcomes (i.e., severe liver fibrosis, decreased response to antivirals, and increased the occurrence of hepatocellular carcinoma) [1]. It is suggested that circulating micro ribonucleic acid (miRNA) can perform regulatory functions to modulate gene expression in peripheral tissues leading to insulin resistance and diabetes. Once miRNA of HCV cleared by treatment, insulin resistance and glucose metabolism improved [2]. A fixed-dose combination of the hepatitis C virus NS5B polymerase inhibitor sofosbuvir, the HCV NS5A inhibitor velpatasvir and the HCV NS3/4A protease inhibitor voxilaprevir is approved in Europe for the treatment of chronic HCV infection in adults [3]. Here we present a case of HCV patient treated with sofosbuvir/velpatasvir/ voxilaprevir that lead to improvement of his hemoglobin A1c (HbA1c) in addition to a significant reduction of his daily insulin dose.

\section{Case Report}

Hepatitis $\mathrm{C}$ virus (HCV) infection is associated with increased rates of glucose abnormalities, including diabetes mellitus and insulin resistance. The presence of glucose abnormalities in HCV infected patients, including diabetes mellitus and insulin resistance, is associated with negative liver-related outcomes (i.e., severe liver fibrosis, decreased response to antivirals, and increased the occurrence of hepatocellular carcinoma) [1]. It is suggested that circulating micro ribonucleic acid (miRNA) can perform regulatory functions to modulate gene expression in peripheral tissues leading to insulin resistance and diabetes. Once miRNA of HCV cleared by treatment, insulin resistance and glucose metabolism improved [2]. 
A fixed-dose combination of the hepatitis C virus NS5B polymerase inhibitor sofosbuvir, the HCV NS5A inhibitor velpatasvir and the HCV NS3/4A protease inhibitor voxilaprevir is approved in Europe for the treatment of chronic HCV infection in adults [3].

Here we present a case of HCV patient treated with sofosbuvir/velpatasvir/voxilaprevir that lead to improvement of his hemoglobin A1c (HbA1c) in addition to a significant reduction of his daily insulin dose (Figure 1). He noticed an improvement in his blood sugar levels after the start of HCV treatment. His HbA1c three months prior to treatment was $7.63 \%$ and after treatment was $7.17 \%$. Concomitantly he reduced the total intake of his insu- lin by $20 \%$. Patient completed his treatment for HCV in October 2017 and became seronegative for HCV on PCR after 12 weeks of therapy with sofosbuvir/velpatasvir/voxilaprevir combination. He stopped using his medications for HCV after 12 weeks. His PCR after HCV treatment is given below in Figure 2. His blood sugar levels and HbA1c before and after HCV Treatment are given Table 1. His recent visit to our center was in April 2018 and latest HbA1c was $7.02 \%$. We noticed a significant improvement in his HbA1c from his pre-HCV treatment value. The patient had not made any significant change in his daily physical activity and diet plan from last one year. He also had not noticed any marked change in his weight.

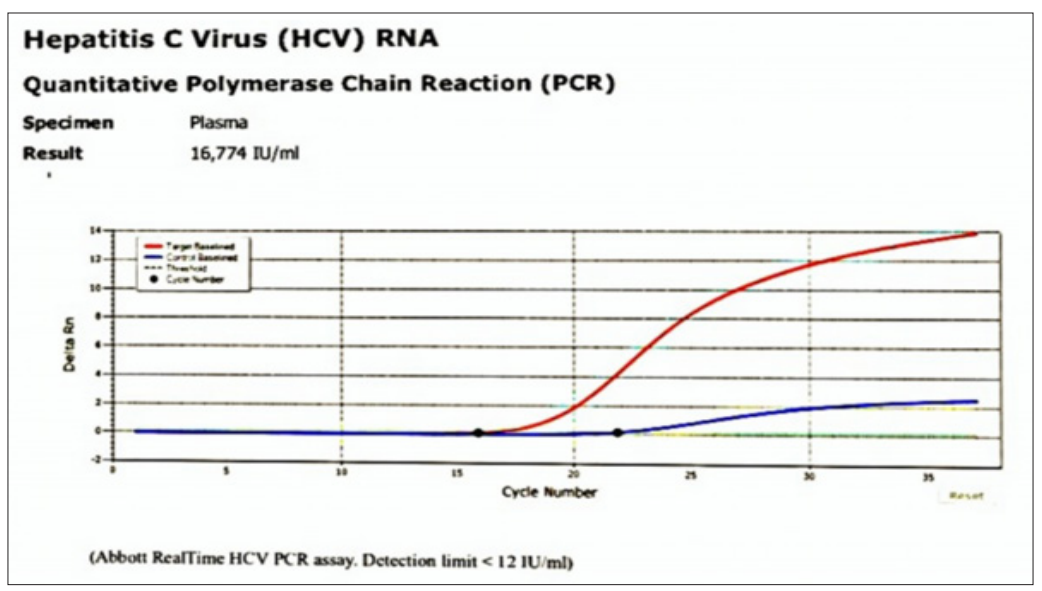

Figure 1: Polymerase chain reaction (PCR) before treatment with sofosbuvir/velpatasvir/voxilaprevir.

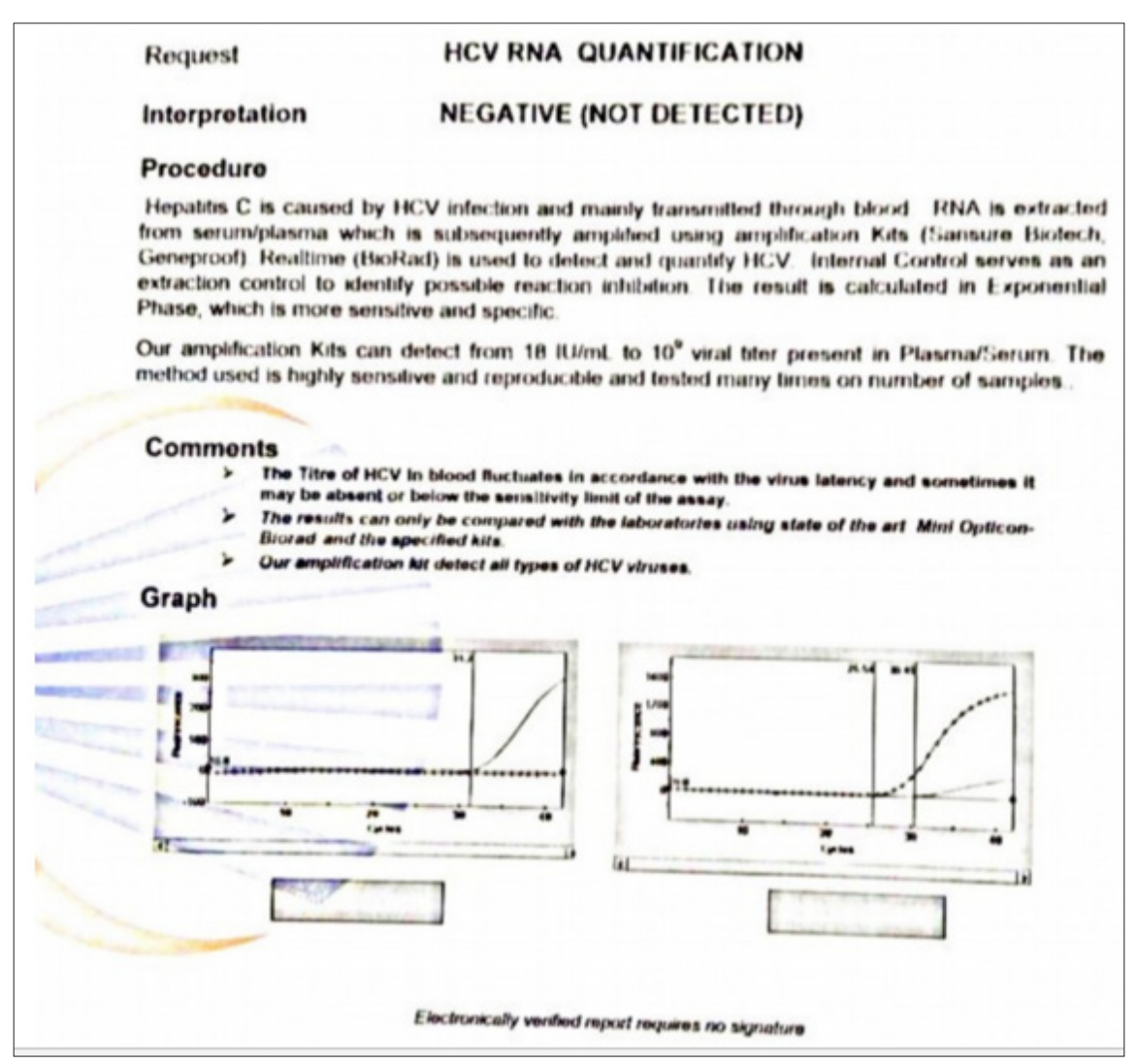

Figure 2: Polymerase chain reaction (PCR) after treatment of HCV with sofosbuvir/velpatasvir/voxilaprevir.

Cite this article: Kashif Aziz, Mehreen Khan, Amir Shahbaz, Muhammad Umair, Issac Sachmechi. Treatment of Hepatitis C with Sofosbuvir, 
Table 1: Laboratory Findings.

\begin{tabular}{|c|c|}
\hline $\begin{array}{c}\text { Before HCV Treatment with } \\
\text { Sofosbuvir/Velpatasvir/ } \\
\text { Voxilaprevir }\end{array}$ & $\begin{array}{c}\text { After HCV Treatment with } \\
\text { sofosbuvir/velpatasvir/ } \\
\text { voxilaprevir }\end{array}$ \\
\hline $\begin{array}{c}\mathrm{HbA} 1 \mathrm{c}=7.63 \%, \text { Blood sugar }=138 \\
\mathrm{gm} / \mathrm{dl}\end{array}$ & $\begin{array}{c}\text { HbA1c }=7.17 \% \text {, Blood sugar level }= \\
120 \mathrm{gm} / \mathrm{dl}\end{array}$ \\
\hline
\end{tabular}

\section{Discussion}

Chronic hepatitis $\mathrm{C}$ virus infection (CHC) is a long-term illness, associated with extrahepatic manifestations, including Type 2 diabetes mellitus (T2DM) which is an endocrine disorder having multifactorial mechanisms. HCV infection is associated with an increased risk of T2DM independent of the severity of the associated liver disease in $\mathrm{CHC}$, and cirrhotic HCV patients. As expected T2DM risk is higher in cirrhotic HCV patients than Chronic Hepatitis C, and the prevalence of HCV infection in T2DM patients is higher than in non-diabetic controls [4]. As we already discussed HCV miRNA role in the development of insulin resistance and diabetes, similarly Antonelli and colleagues [5] also explained the processes through which $\mathrm{CHC}$ is associated with T2DM seem to involve direct viral effects, insulin resistance, proinflammatory cytokines, chemokines, and other immune-mediated mechanisms. It is suggested that Clearance of HCV with direct-acting antivirals (DAAs) reported improvements in glucose metabolism and decreases HbA1c, as well as dependence on anti-diabetic medications $[5,6]$.

As in our case patient reduced his dose of insulin by $20 \%$ after HCV treatment. Also, as we already mentioned our patient had not made any significant change in his diet plan and daily physical activity during last one year, so we can conclude that improvement in his glucose metabolism is related to HCV eradication. On review of the literature, we found four studies which show improvement in HbA1c and insulin resistance after the treatment of HCV by sofosbuvir with ledipasvir and other DAAs as shown below Table 2. One of these studies also shows decrease dependence on antiglycemic medication after HCV eradication. Our patient is the first case to be reported which shows improvement in HbA1c, as well as the reduction in daily insulin dose due to HCV treatment with sofosbuvir/velpatasvir/voxilaprevir.

Table 2: Review of Literature.

\begin{tabular}{|c|c|c|c|c|}
\hline Authors & Study type & Aim of study & Summary of study & Study outcome \\
\hline Hum et al. [6] & Retrospective study & $\begin{array}{l}\text { The aim of the study was } \\
\text { to investigate whether } \\
\text { elimination of hepatitis C } \\
\text { (HCV) infection with direct- } \\
\text { acting antiviral (DAAs) agents } \\
\text { is associated with improved } \\
\text { blood glucose control in } \\
\text { diabetic patients. }\end{array}$ & $\begin{array}{l}\text { They identified 2,435 patients with } \\
\text { diabetes who underwent interferon-free } \\
\text { and ribavirin-free DAAs-based antiviral } \\
\text { treatment for HCV. Changes in average } \\
\text { hemoglobin A1c (HbA1c) level and use } \\
\text { of antidiabetic medications one year } \\
\text { before and after antiviral treatment } \\
\text { were compared between patients who } \\
\text { achieved Sustained Virologic Response } \\
\text { (SVR) and those who did not. }\end{array}$ & $\begin{array}{l}\text { DAAs-based eradication of HCV } \\
\text { is associated with an improved } \\
\text { glycemic control in patients } \\
\text { with diabetes as evidenced by } \\
\text { decreased mean HbA1c and } \\
\text { decreased insulin use. These } \\
\text { endocrine benefits of SVR } \\
\text { provide additional justification } \\
\text { for considering antiviral } \\
\text { treatment in all patients with } \\
\text { diabetes. }\end{array}$ \\
\hline Ikeda et al. [7] & Observational study & $\begin{array}{l}\text { The aim of the study was to } \\
\text { evaluate whether the DAAs } \\
\text { treatment without ribavirin } \\
\text { lowers HbA1c at } 12 \text { weeks } \\
\text { after therapy completion. }\end{array}$ & $\begin{array}{l}\text { An observational study to assess the } \\
\text { effect of sofosbuvir and ledipasvir (SOF/ } \\
\text { LED) treatment on glycemic control. } \\
\text { Patients with HCV eradication, HbA1c } \\
\text { levels decreased significantly after } \\
\text { treatment. }\end{array}$ & $\begin{array}{l}\text { They concluded that hepatitis } \\
\text { C eradication by SOF/LED was } \\
\text { associated with an improvement } \\
\text { of glucose metabolism in the } \\
\text { population with or without } \\
\text { diabetes and suggests further } \\
\text { investigation. }\end{array}$ \\
\hline $\begin{array}{l}\text { Morales, et } \\
\text { al. [8] }\end{array}$ & $\begin{array}{l}\text { Retrospective } \\
\text { analysis }\end{array}$ & $\begin{array}{l}\text { The aim of the study was to } \\
\text { assess the effect of SOF based } \\
\text { regimens on glycemic and } \\
\text { lipid control. }\end{array}$ & $\begin{array}{l}\text { They evaluated the changes in glucose } \\
\text { and lipid metabolism in a group of } \\
\text { hepatitis C patients treated and cured } \\
\text { with a sofosbuvir-containing regimen } \\
\text { [SOF/ribavirin/interferon, SOF/ } \\
\text { simeprevir, or SOF/ledipasvir (LDV) } \pm \\
\text { ribavirin]. Patients with HbA1c and lipid } \\
\text { panels within six months before and six } \\
\text { months after therapy were identified and } \\
\text { included in this study. }\end{array}$ & $\begin{array}{l}\text { Eradication of HCV with a SOF } \\
\text { regimen resulted in a significant } \\
\text { drop in HbA1c and an increase } \\
\text { in LDL and Total cholesterol post } \\
\text { therapy. }\end{array}$ \\
\hline $\begin{array}{c}\text { Pavone, et al. } \\
\text { [9] }\end{array}$ & $\begin{array}{l}\text { Retrospective } \\
\text { analysis }\end{array}$ & $\begin{array}{l}\text { The aim of this study was to } \\
\text { evaluate the glycaemic control } \\
\text { modifications in a case series } \\
\text { of HCV-positive diabetic } \\
\text { patients receiving direct- } \\
\text { acting antiviral. }\end{array}$ & $\begin{array}{l}\text { They evaluated } 149 \mathrm{HCV} \text {-positive } \\
\text { patients. They were able to identify } 29 \\
\text { patients with type } 2 \text { diabetes mellitus } \\
\text { who were receiving different interferon- } \\
\text { free regimens. During treatment, the } \\
\text { analysis revealed a statically significant } \\
\text { reduction in Fasting glucose and HbA1c } \\
\text { levels. }\end{array}$ & $\begin{array}{l}\text { Treatment of HCV Patients } \\
\text { with DAAs results in marked } \\
\text { reductions in fasting glucose } \\
\text { and HbA1c. Fasting Glucose and } \\
\text { HbA1c reductions values were } \\
\text { independent of which DAAs was } \\
\text { present in the regimen, HCV } \\
\text { genotype, and body mass index. }\end{array}$ \\
\hline
\end{tabular}




\section{Conclusion}

HCV eradication by direct acting antivirals like sofosbuvir/ velpatasvir/voxilaprevir was accompanied by the improvement in $\mathrm{HbA1c}$ with reduction of the daily dose of insulin. We conclude that the elimination of miRNA of HCV by direct acting antiviral is one of the reasons for the improvement in the glucose metabolism. Future studies are needed to confirm the association between the glycemic level and HCV eradication by direct acting antivirals.

\section{Protection of Humans and Animals}

The authors declare that the procedures were followed according to the regulations established by the Clinical Research and Ethics Committee and to the Helsinki Declaration of the World Medical Association.

\section{Data Confidentiality}

The authors declare having followed the protocols in use at their working center regarding patients' data publication.

\section{Patient Consent}

Obtained.

\section{References}

1. Desbois AC, Cacoub P (2017) Diabetes mellitus, insulin resistance and hepatitis C virus infection. A contemporary review. World J Gastroenterol 23(9): 1697-1711.

\section{ISSN: 2574-1241}

DOI: $10.26717 / B J S T R .2018 .09 .001792$

Kashif Aziz. Biomed J Sci \& Tech Res

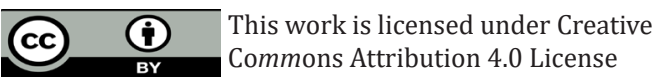

Submission Link: https://biomedres.us/submit-manuscript.php
2. Singhal A, Agrawal A, Ling J (2018) Regulation of insulin resistance and type II diabetes by Hepatitis $\mathrm{C}$ virus infection: A driver function of circulating miRNAs. J Cell Mol Med 22(4): 2071-2085.

3. Heo YA, Emma D Deeks (2018) Sofosbuvir/Velpatasvir/Voxilaprevir: A Review in Chronic Hepatitis C. Drugs 78(5): 577-587.

4. Fabiani S, Fallahi P, Ferrari SM, Miccoli M, Antonelli A (2018) Hepatitis C virus infection and development of type 2 diabetes mellitus: Systematic review and meta-analysis of the literature. Rev Endocr Metab Disord.

5. Antonelli A, Ferrari SM, Giuggioli D, Andrea Di Domenicantonio, Ilaria Ruffilli, et al. (2014) Hepatitis C virus infection and type 1 and type 2 diabetes Mellitius. World J Diabetes 5(5): 586-600.

6. Hum J, Jou JH, Green PK, Berry K, Lundblad J, et al. (2017) Improvement in glycemic control of type 2 diabetes after successful treatment of hepatitis C virus. Diabetes Care 40: 1173-1180.

7. Ikeda A, Ikeda K, Takai A, Takahashi K, Ueda, et al. (2017) Hepatitis C Treatment with Sofosbuvir and Ledipasvir accompanied by Immediate Improvement in Hemoglobin A1c. Digestion 96(4): 228-230.

8. Morales AL, Junga Z, Singla MB, Sjogren M, Torres D (2016) Hepatitis $C$ eradication with Sofosbuvir leads to significant metabolic changes. World J Hepatol 8(35): 1557-1563.

9. Pavone P, Tieghi T, D'Ettorre G, Lichtner M, Marocco R, et al. (2016) Rapid decline of fasting glucose in HCV diabetic patients treated with direct-acting antiviral agents. Clin Microbiol Infect 22(5): 462.

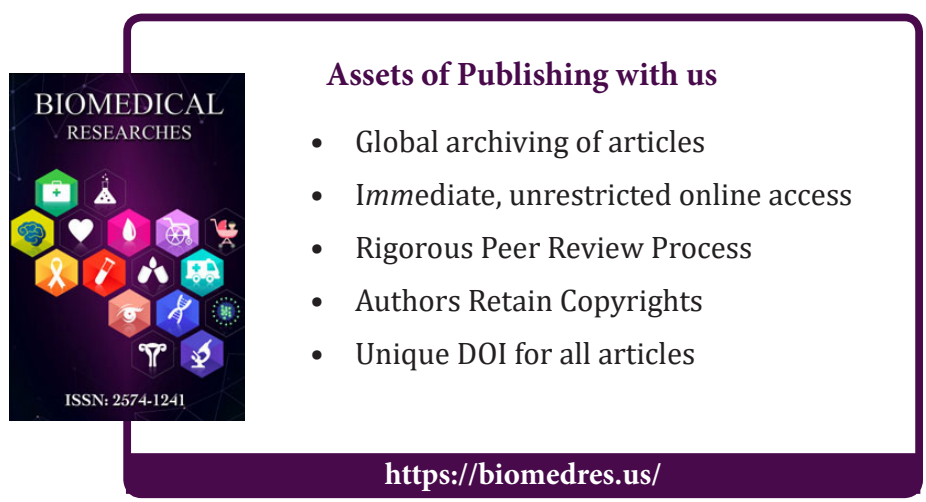

